\title{
HIERARCHICAL CONTROL FOR A CLASS OF UNCERTAIN PIECEWISE LINEAR HYBRID DYNAMICAL SYSTEMS
}

\author{
Hai Lin ${ }^{*, 1}$ Xenofon D. Koutsoukos ${ }^{* *}$ \\ Panos J. Antsaklis *,1 \\ * EE. Dept. Univ. of Notre Dame, Notre Dame IN-46556, USA \\ ** Xerox Palo Alto Research Center, Palo Alto, CA 94304, USA
}

\begin{abstract}
In this paper, we consider the hierarchical control problem for a class of uncertain hybrid dynamical systems. The continuous dynamics of this class of uncertain hybrid systems are described by linear difference state equations, whose right side functions are unknown but lie within some convex hulls of known functions. Our control objective is for the closed loop system to exhibit a desired behavior under the dynamic uncertainty, continuous disturbances and uncontrollable events. One of the main questions is the existence of appropriate controllers. We will focus on this question here, and present a novel methodology for the analysis of uncertain piecewise linear hybrid dynamical systems (PLHDS) based on backward reachability analysis. For this purpose, we derive the predecessor operator for this class of uncertain PLHDS. Then both static control specifications such as safety and reachability, and dynamic control specifications are considered. A temperature control system is used for illustration.
\end{abstract}

Keywords: Hybrid Systems, Piecewise Linear Systems, Hierarchical Control, Uncertainty

\section{INTRODUCTION}

In this paper, a novel methodology for the analysis of uncertain piecewise linear hybrid dynamical systems based on backward reachability analysis is presented. Piecewise linear systems arise as mathematical models of systems in many practical applications, often from linearization of nonlinear systems (c.f. (Sontag, 1996; Johansson, 1999; Koutsoukos, 2000b)). However, for a large class of systems, like systems with uncertainty or parameter variations, or systems with strong nonlinearities, parameters variations have to be considered otherwise the operating vicinity of the linearization has to be reduced, then increasing the number of linearizations, which is not prac-

1 The partial support of the National Science Foundation (NSF ECS99-12458 \& CCR01-13131), and of the DARPA/ITO-NEST Program (AF-F30602-01-2-0526) is gratefully acknowledged. tical in many cases. Therefore it is important to study the class of uncertain, or parameter-variant piecewise linear systems. In (Johansson, 1999), computational algorithms for the analysis of nonlinear and uncertain dynamical systems based on the piecewise quadratic Lyapunov functions and convex optimization are developed. In (Hespanha et al., 2001), the problem of controlling a poorly modelled continuous-time linear system is addressed. The proposed approach is to employ logic-based switching among a family of candidate controllers.

The work presented here is an extension of our group's recent work in the field of Hierarchical Control of Hybrid Dynamical Systems (Koutsoukos and Antsaklis, 2001) ${ }^{2}$. The control objective of the hierarchical control problem is for

\footnotetext{
2 It is related to supervisory control framework for hybrid
} systems (cf. (Stiver et al., 1996; Raisch and O'Young, 1998; 
the closed loop system to follow a desired output which is assumed to be generated by another discrete dynamical system. This framework leads naturally to an input-output representation of the constituent systems which is analogous to classical control design. One of the main questions here is the existence of the appropriate controller. In this paper we consider uncertain piecewise linear hybrid dynamical systems described in definition 1 , where the continuous dynamics matrix $\tilde{A}_{q}$ is unknown but can be written as a convex combination of known matrices. And a methodology based on the predecessor operator and backward reachability analysis (Section 3) for analysis of such uncertain piecewise linear hybrid dynamical systems is presented.

The structure of this paper is as follows. Section 2 defines a class of uncertain piecewise linear hybrid dynamical systems. Section 3 considers the predecessor operator and backward reachability analysis. Section 4 deals with static control specifications, such as safety and reachability. The dynamical control specifications are considered in Section 5, and an example is given. Then we conclude with some discussion about future work.

\section{PROBLEM FORMULATION}

In the following, we define a class of piecewise linear hybrid dynamical systems with uncertainty, in which the continuous dynamics matrix $\tilde{A}_{q}$ is unknown but can be written as a convex combination of a finite number of known matrices of proper dimensions. The discrete dynamics are described by finite automata, and the interaction between the continuous and the discrete part is defined by piecewise linear maps. The exact definition is as follows.

Definition 1. Consider the Uncertain Piecewise Linear Hybrid Dynamical Systems (uncertain PLHDS) defined by

$$
\begin{aligned}
x(t+1) & =\tilde{A}_{q(t)} x(t)+B_{q(t)} u(t)+E_{q(t)} d(t) \\
q(t+1) & =\delta\left(q(t), \pi(x(t)), \sigma_{c}(t), \sigma_{u}(t)\right) \\
y(t) & =g(q(t), x(t))
\end{aligned}
$$

where $x(0)=x_{0} \in X \subset \mathbb{R}^{n} ; q(0)=q_{0} \in Q, X$ and $Q$ stand for the continuous state space and collection of discrete states (modes) respectively. $u \in \mathcal{U} \subset \mathbb{R}^{m}, d \in \mathcal{D} \subset \mathbb{R}^{p}$, and $\mathcal{U}, \mathcal{D}$ are bounded polyhedral set. And

- $\tilde{A}_{q} \in \mathbb{R}^{n \times n}$. The entries in $\tilde{A}_{q}$ are unknown, but $\tilde{A}_{q}$ can be expressed as a convex combination of $N_{q} \mathbb{R}^{n \times n}$ matrices $\left\{A_{q}^{1}, A_{q}^{2}, \ldots, A_{q}^{N_{q}}\right\}$, that is

$$
\tilde{A}_{q}=\sum_{i=1}^{N_{q}} \lambda_{i} A_{q}^{i}, \quad \lambda_{i} \geq 0, \sum_{i=1}^{N_{q}} \lambda_{i}=1
$$

Koutsoukos et al., 2000a)). For the relationship of the two methods, please see for example (Koutsoukos, 2000b).
- $B_{q} \in \mathbb{R}^{n \times m}$, and $E_{q} \in \mathbb{R}^{n \times p}$ are the system matrices for the discrete state $q$,

- $\pi: X \rightarrow X / E_{\pi}$ partitions the continuous state space $\mathbb{R}^{n}$ into polyhedral equivalence classes. ${ }^{3}$

- $q(t+1) \in \operatorname{act}(\pi(x(t)))$, where $a c t: X / E_{\pi} \rightarrow 2^{Q}$ defines the active mode set,

- $\delta: Q \times X / E_{\pi} \times \Sigma_{c} \times \Sigma_{u} \rightarrow Q$ is the discrete state transition function. Here $\sigma_{c} \in \Sigma_{c}$ denotes a controllable event and $\Sigma_{u}$ the collection of uncontrollable events.

- $g: Q \times X \rightarrow Y$ is the output function which is assumed to be piecewise linear.

Assume that the current discrete state is $q$ and that $q^{\prime} \in \operatorname{act}(\pi(x(t)))$ for some state $x(t) \in \mathbb{R}^{n}$, then $q^{\prime}$ is a possible new state, and the transition $q \rightarrow q^{\prime}$ (or $\left.\left(q, q^{\prime}\right)\right)$ may occur. The guard $G\left(q, q^{\prime}\right)$ of the transition $\left(q, q^{\prime}\right)$ is defined as the set of all states $(q, x)$ such that $q^{\prime} \in \operatorname{act}(\pi(x(t)))$ and there exist controllable event $\sigma_{c} \in \Sigma_{c}$ such that $q^{\prime}=\delta\left(q, \pi(x), \sigma_{c}, \sigma_{u}\right)$ for every uncontrollable event $\sigma_{u} \in \Sigma_{u}$. The guard of the transition describes the region of the hybrid state space where the transition can be forced to take place independently of the disturbances generated by the environment.

Remark: For the case of parameter variations, i.e. $\tilde{A}_{q} \in\left\{A_{q} \in \mathbb{R}^{n \times n}: a_{i j} \in\left[\bar{a}_{i j}, \underline{a}_{i j}\right]\right\}, \tilde{A}_{q}$ can always be written as convex combination of finite number $^{4}$ of corner matrices, i.e. $a_{i j} \in\left\{\bar{a}_{i j}, \underline{a}_{i j}\right\}$.

\section{BACKWARD REACHABILITY ANALYSIS}

The main mathematical tool to be used for backward reachability analysis is the predecessor operator applied recursively to subsets of the hybrid state space. The application of the predecessor operator corresponds to a partition refinement into finer partitions that allow the formulation of conditions that guarantee the existence of appropriate controls for the objectives of interest.

\subsection{The Predecessor Operator}

A region of the state space is defined as $R \subset Q \times$ $X$. We are interested in computing the set of all the states that can be driven to $R$ by either continuous or discrete transitions. In the case of piecewise linear hybrid dynamical systems, it suffices to assume that the region is represented by $R=(q, P)$ where $q \in Q$ and $P \subset \mathbb{R}^{n}$ is a piecewise linear set. Please note the fact that piecewiselinear algebra admits elimination of quantifiers (Sontag, 1982), which implies that a piecewise linear set can always be represented by using only

\footnotetext{
3 This is called as primary partition, which is designed based on the specifications and the continuous dynamics. ${ }^{4}$ Here $N_{q}=2^{n^{2}}$ in general, but if we can represent all $\left(A_{q}, B_{q}\right)$ in controller form, then $N_{q}$ will be reduced to $2^{n}$.
} 
linear equalities and inequalities. So let's assume that $P$ can be represented by $P=\left\{x \in \mathbb{R}^{n} \mid G x \leq\right.$ $w\}^{5}$.

The dynamic evolution of the system is defined by discrete and continuous transitions. We first define and compute the predecessor operator for discrete transitions.

\section{Discrete Transitions}

The predecessor operator for discrete transitions is denoted by pre $_{d}: 2^{Q \times X} \rightarrow 2^{Q \times X}$ and it is used to compute the set of states that can be driven to the region $R$ by a discrete instantaneous transition $q^{\prime} \rightarrow q$ that can be forced by the controller for any uncontrollable event. The predecessor operator in this case is defined as follows:

$$
\begin{array}{r}
\text { pre }_{d}(R)=\left\{\left(q^{\prime}, x\right) \in Q \times X \mid \exists \sigma_{c} \in \Sigma_{c}, \forall \sigma_{u} \in \Sigma_{u},\right. \\
\left.q=\delta\left(q^{\prime}, x, \sigma_{c}, \sigma_{u}\right)\right\}
\end{array}
$$

For every discrete transition that can be forced by a controllable event we have that

$$
\operatorname{pre}_{d}(R)=\bigcup_{q^{\prime} \in \operatorname{act}(P)} G\left(q^{\prime}, q\right)
$$

where $G\left(q^{\prime}, q\right)$ is the guard of transition $q^{\prime} \rightarrow q$.

\section{Continuous Transitions}

In the case of continuous transitions, given the region $R=(q, P)$ we define the predecessor operator pre $_{c}: 2^{Q \times X} \rightarrow 2^{Q \times X}$ to compute the set of states for which there exists a control input so that the continuous state will be driven in the set $P$ for every disturbance, while the system is at the discrete mode $q$. The action of the operator is described by

$$
\begin{aligned}
& \operatorname{pre}_{c}^{q}(R)=\{q\} \times\{x \in X \mid \exists u \in \mathcal{U}, \forall d \in \mathcal{D}, \\
&\left.\forall \tilde{A}_{q} \in \operatorname{Conv}_{i=1}^{N_{q}}\left(A_{q}^{i}\right), \quad \tilde{A}_{q} x+B_{q} u+E_{q} d \in P\right\}
\end{aligned}
$$

\section{Computation of the Predecessor Operator}

As it was explained above, the predecessor operator for discrete transitions is given by the union of the guards of those transitions that are feasible and can be forced by a control mechanism. Since the guards are regions of the state space that are included in the description of the primary partition, here we concentrate on predecessor operator for the continuous transitions.

Let's denote $\operatorname{pre}_{c, i}^{q}(R)$ for $1 \leq i \leq N_{q}$ as $\operatorname{pre}_{c, i}^{q}(R)=\{q\} \times\left\{x \in X \mid \exists u \in \mathcal{U}, \forall d \in \mathcal{D}, A_{q}^{i} x+\right.$ $\left.B_{q} u+E_{q} d \in P\right\}^{6}$, and assume that the piecewise linear set $P=\left\{x \in \mathbb{R}^{n} \mid G x \leq w\right\}$, where $G \in \mathbb{R}^{v \times n}, w \in \mathbb{R}^{v}$. Then, we have a proposition:

\footnotetext{
5 Here $a \leq b$ means that all entries in the vector $(a-b)$ are all non-positive.

6 The $\operatorname{pre}_{c, i}^{q}(R)$ is nothing but continuous predecessor set of the $i$-th vertex $A_{q}^{i}$.
}

Proposition 2.

$$
\operatorname{pre}_{c}^{q}(R)=\bigcap_{i=1, \ldots, N_{q}} \operatorname{pre}_{c, i}^{q}(R)
$$

Proof: Firstly, from definition of $\operatorname{pre}_{c}^{q}(R)$ it is quite straight forward to show that

$$
\operatorname{pre}_{c}^{q}(R) \subseteq \bigcap_{i=1, \ldots, N_{q}} \operatorname{pre}_{c, i}^{q}(R)
$$

Because for all $i=1, \ldots, N_{q}, A_{q}^{i} \in \operatorname{Co}_{i=1}^{N_{q}}\left(A_{q}^{i}\right)$, by definition $\operatorname{pre}_{c}^{q}(R) \subseteq \operatorname{pre}_{c, i}^{q}(R)$, for all $i$, so $\operatorname{pre}_{c}^{q}(R) \subseteq \bigcap_{i=1, \ldots, N_{q}} \operatorname{pre}_{c, i}^{q}(R)$.

On the other hand, for any state $(q, x) \in$ $\bigcap_{i=1, \ldots, N_{q}} \operatorname{pre}_{c, i}^{q}(R)$, by definition we have

$$
\begin{aligned}
\forall d \in \mathcal{D}, \forall 1 \leq i \leq N_{q}, \exists u \in \mathcal{U}, \text { s.t. } \\
\qquad A_{q}^{i} x+G B_{q} u+G E_{q} d \leq w
\end{aligned}
$$

which is equivalent to

$\forall d \in \mathcal{D}, \forall 1 \leq i \leq N_{q}, \forall 1 \leq j \leq v, \exists u \in \mathcal{U}$, s.t.

$$
g_{j}^{T} A_{q}^{i} x+g_{j}^{T} B_{q} u+g_{j}^{T} E_{q} d \leq w_{j}
$$

where $g_{j}^{T}$ denotes the $j$-th row vector of matrix $G$. Consider function of $\lambda=\left[\lambda_{1}, \lambda_{2}, \ldots, \lambda_{N_{q}}\right]^{T}$, $g_{j}^{T} \sum_{i=1}^{N_{q}}\left(\lambda_{i} A_{q}^{i}\right) x$, which is linear in $\lambda$ and the variable $\lambda$ is within a closed set, so $\max _{\lambda}\left(g_{j}^{T} \sum_{i=1}^{N_{q}}\left(\lambda_{i} A_{q}^{i}\right) x\right)$ is attainable and can only occur at the boundary, i.e. vertex, $A_{q}^{i}$, in this case. That is, for $\forall 1 \leq j \leq v$ there always exist $i_{j}$, such that $\max _{\lambda}\left(g_{j}^{T} \sum_{i=1}^{N_{q}}\left(\lambda_{i} A_{q}^{i}\right) x\right)=g_{j}^{T} A_{q}^{i_{j}} x$, so

$\forall d \in \mathcal{D}, \forall 1 \leq j \leq v, \forall \lambda_{i} \in[0,1], \sum_{i=1}^{N_{q}}\left(\lambda_{i}\right)=1, \exists u \in \mathcal{U}$,

$$
\begin{aligned}
& \text { s.t. } \quad g_{j}^{T} \sum_{i=1}^{N_{q}}\left(\lambda_{i} A_{q}^{i}\right) x+g_{j}^{T} B_{q} u+g_{j}^{T} E_{q} d \\
& \leq \max _{\lambda}\left(g_{j}^{T} \sum_{i=1}^{N_{q}}\left(\lambda_{i} A_{q}^{i}\right) x\right)+g_{j}^{T} B_{q} u+g_{j}^{T} E_{q} d \\
& =g_{j}^{T} A_{q}^{i_{j}} x+g_{j}^{T} B_{q} u+g_{j}^{T} E_{q} d \leq w_{j}
\end{aligned}
$$

Therefore,

$$
\begin{aligned}
\forall d \in \mathcal{D}, \forall \lambda_{i} \in[0,1], \sum_{i=1}^{N_{q}}\left(\lambda_{i}\right)=1, \exists u \in \mathcal{U}, \text { s.t. } \\
G \sum_{i=1}^{N_{q}}\left(\lambda_{i} A_{q}^{i}\right) x+G B_{q} u+G E_{q} d \leq w
\end{aligned}
$$

Then, by definition of $\operatorname{pre}_{c}^{q}(R)$, we conclude $(q, x) \in \operatorname{pre}_{c}^{q}(R)$. Then

$$
\bigcap_{i=1, \ldots, N_{q}} \operatorname{pre}_{c, i}^{q}(R) \subseteq \operatorname{pre}_{c}^{q}(R)
$$

Then we conclude

$$
\operatorname{pre}_{c}^{q}(R)=\bigcap_{i=1, \ldots, N_{q}} \operatorname{pre}_{c, i}^{q}(R)
$$


Remark: $\operatorname{pre}_{c, i}^{q}(R)$ is given by $\operatorname{pre}_{c, i}^{q}(R)=$ $\operatorname{proj}(S)$ (Koutsoukos, 2000b; Koutsoukos and Antsaklis, 2001) ${ }^{7}$, where $S \subseteq X \times \mathcal{U}$ is defined as

$$
S=\left\{(x, u) \mid \bigwedge_{j=1, \ldots, v}\left(g_{j}^{T} A_{q}^{i} x+g_{j}^{T} B u \leq w_{j}-g_{j}^{T} E d_{j}^{*}\right\}\right.
$$

where, $d_{j}^{*}=\arg \max _{d \in \mathcal{D}} g_{j}^{T} E d$.

Remark: Please note that $\operatorname{pre}_{c, i}^{q}(R)$ is piecewise linear $(\mathrm{PL})$ and $\mathrm{PL}$ set is closed under finite intersection, so $\bigcap_{i=1}^{N_{q}} \operatorname{pre}_{c, i}^{q}(R)$ is also PL set and comparably easy to be calculated and efficiently represented. In the following sections we will derive necessary and sufficient conditions for safety, reachability and attainability problems based on this proposition.

\subsection{Algorithms for Backward Reachability Analysis} Consider an uncertain PLHDS and a region $R=$ $(q, P)$. We denote the partition of the continuous state space $X$ as $\{P i\} ; i=1, \ldots|\pi|$. The following algorithm computes all the states of the hybrid system that can be driven to $R$ in one time-step.

\section{Algorithm 1. Predecessor Operator}

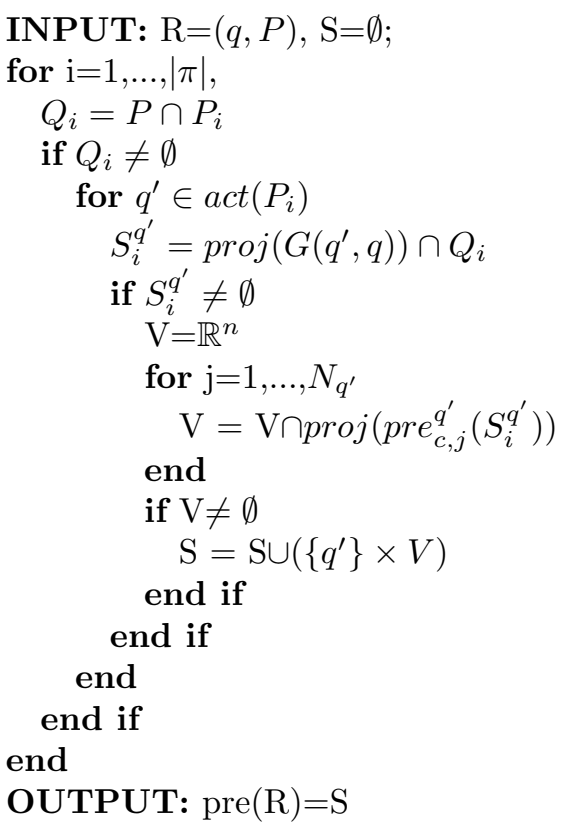

Remark: Please note that the set $\operatorname{pre}(R)$ is piecewise linear and is described using a finite set of linear inequalities. Therefore, we can apply the predecessor operator to compute the set of all states that can be driven to $\operatorname{pre}(R)$ to get $\operatorname{pre}(\operatorname{pre}(R))$. Following the same procedure, we define successive applications of the predecessor operator as:

$$
\operatorname{pre}^{M}(R)=\underbrace{\operatorname{pre}(\ldots \text { pre }(R))}_{\text {Mtimes }}
$$

\footnotetext{
$7 \operatorname{proj}(q \times X)=X$, This is accomplished efficiently by Fourier-Motzkin elimination and linear programming techniques (Motzkin, 1952).
}

Remark: For a given region $\mathrm{R}$, we define the coreachable set $C R(R)$ as the set of all states that can be driven to $\mathrm{R}$. The coreachable set for a region of the hybrid state space can be computed by successive application of the predecessor operator

$$
C R(R)=\operatorname{pre}^{*}(R)
$$

In general, the proposed procedure is semidecidable and its termination is not guaranteed. We will return to this matter in the reachability problem shortly after.

\section{SAFETY AND REACHABILITY}

Typical control specifications investigated in this paper are formulated in terms of partitions of the state space of the system. Examples include safety problems, where the controller guarantees that the plant will not enter an unsafe region.

\section{Safety}

At first, we focus on the safety problem and we show how the refinement of the state space partition can be used to formulated conditions for safety. Given a set of states described by the region $R \subset Q \times X$ and an initial condition $\left(q_{0}, x_{0}\right) \in R$,we say that the system is safe if $(q(t), x(t)) \in R$ for every $t \geq t_{0}$. The conditions that guarantee that a given region of the hybrid state space is safe can be described as following ${ }^{8}$.

Theorem 3. An uncertain PLHDS is safe with respect to the region $R \subseteq Q \times X$ if and only if $R \subseteq \operatorname{pre}(R)$.

This safety condition can be efficiently tested by solving a finite number of linear programming problems that depends on the number of regions and discrete states of the system.

\section{Reachability}

Secondly, we study the reachability problem for uncertain piecewise linear hybrid dynamical systems. It should be emphasized that we are interested only in the case when reachability between two regions $R_{1}$ and $R_{2}$ is defined so that the state is driven to $R_{2}$ directly from the region $R_{1}$ in finite steps without entering a third region. We only consider regions of the form $R_{1}=\left(Q_{1}, P_{1}\right)$ and $R_{2}=\left(Q_{2}, P_{2}\right)$ for which $P_{1}$ and $P_{2}$ are adjacent polyhedral regions of the primary partition. This is a problem of practical importance in hybrid systems since it is often desirable to drive the state to a target region of the state space while satisfying constraints on the state and input during the operation of the system.

The problem of deciding whether a region $R_{2}$ is directly reachable from $R_{1}$ can be solved by

\footnotetext{
8 The proof is analogous to proof for the corresponding theorem on (Koutsoukos and Antsaklis, 2001; Koutsoukos, 2000b).
} 
recursively computing all the states that can be driven to $R_{2}$ from $R_{1}$ using the predecessor operator. As we have discussed, the proposed procedure is semi-decidable and its termination is not guaranteed. In order to formulate a constructive algorithm for reachability, we consider two approaches. First, we consider an upper bound on the time horizon and we examine the reachability only for the predetermined finite horizon. Second, we formulate a termination condition for the reachability algorithm based on a grid-based approximation of the piecewise linear regions of the state space (Koutsoukos and Antsaklis, 2001).

Theorem 4. ${ }^{8}$ Consider an uncertain PLHDS described by definition 3 and the regions $R_{1}=$ $\left(Q_{1}, P_{1}\right)$ and $R_{2}=\left(Q_{2}, P_{2}\right)$. if and only if $R_{1} \subseteq$ $C R\left(R_{2}\right)^{9}$ then the region $R_{2}$ is directly reachable from $R_{1}$.

\section{EXOSYSTEM AND ATTAINABILITY}

In the previous section, we dealt with safety and reachability. They are called as static specifications that do not change as time progresses. Here, we will turn to dynamic specifications that involve sequencing of events and eventual execution of actions.

In general, a regulator requests certain types of outputs from the plant so that these are attained in the presence of disturbances. The desired outputs can be described as the outputs of another dynamical system, called the exosystem. We model the control specifications using an inputoutput deterministic finite automaton. We are interested in either static specifications that do not change as time progresses or dynamic specifications that include, for example, sequencing of events. The dynamic behavior of the exosystem is described by the set of output sequences it can generate.

\section{Dynamic Control Specifications}

In this section, we present a modeling formalism for control specifications based on finite automata models, and we consider dynamic specifications. The main advantages of the formal modeling of the specifications are the following. First, formal modeling allows to compose complex specifications from simpler ones. The controller synthesis relies directly on the finite automaton model of the specifications. Therefore, by putting the emphasis on the specifications, we can develop a systematic methodology for controller synthesis. Finally, the formal models of the specifications are necessary for simulation and verification tools.

${ }^{9} C R\left(R_{2}\right)$ can be calculated by $C R^{N}\left(R_{2}\right)$ or by grid-based approximation.
As it was discussed, the primary partition is designed based on the specifications. We consider specifications that are described with respect to regions of the hybrid state space. We define the set $X_{e}$ as $X_{e}=\left\{R_{1}, R_{2}, \ldots, R_{M}\right\}$ where $R_{i}=\left(Q_{i}, P_{i}\right)$ are piecewise linear regions of the hybrid state space. Since we assume that the primary partition is fine enough to describe the specifications, for every region we can write $R_{i} \subseteq Q \times X / E_{\pi}$. In the following, we use a formal automaton model to represent the specifications of interest.

Definition 5. The control specifications are modelled by an input-output (I/O) deterministic finite automaton described by $\mathcal{E}=\left(X_{e}, V_{e}, Y_{e}, \delta_{e}, \lambda_{e}, R_{0}\right)$ where $X_{e}$ is the set of states, $V_{e}$ is the input alphabet, $Y_{e}$ is the output alphabet, $\delta_{e}: X_{e} \times V_{e} \rightarrow X_{e}$ is the state transition function, $\lambda_{e}: X_{e} \rightarrow Y_{e}$ is the output function returning the output associated with each state, and $R_{0}$ is the initial state.

We assume that the function $\delta_{e}$ is non-total, which means that not every input can be applied to every state of the automaton. We also assume that every state is reachable and therefore, there exists appropriate input sequences so that every state can be reached. The I/O finite automaton which describes the specifications is a deterministic Moore automaton and is called the exosystem.

\section{Attainability}

Our control objective is that the closed loop system consisting of the plant and the controller exhibits the same behavior as the exosystem. And the main question is if there exists a controller so that the closed loop system follows the behavior of the exosystem. We formalize this notion using the attainability of the specified behavior. In the following we present the necessary and sufficient condition for attainability. In this work, attainable behavior refers to behavior that can be forced to the plant by a control mechanism.

Theorem 6. (Koutsoukos and Antsaklis, 2001) The specification behavior $B_{s p}$ is attainable if and only if the following conditions hold:

First, Every terminating state $y_{n}$ corresponds to a region $R_{n}$ that is safe; and secondly for every nonterminating state $y_{k}$, there exists $y_{k+1}$ so that, for the corresponding regions we have that $R_{k+1}$ is reachable from $R_{k}$.

Furthermore, if $B_{s p}$ is attainable then there exists a controller $\mathcal{C}$ so that the regulator problem has a solution.

To illustrate the idea, We present a temperature control system as an example (Koutsoukos and Antsaklis, 2001).

Example 7. (Temperature Control System). The system consists of a furnace that can be switched on and off. The control objective is to control the temperature at a point of the system 


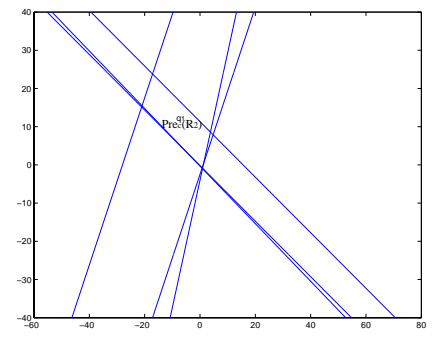

Fig. 1. Illustration for the predecessor set $\operatorname{pre}_{c}^{q_{1}}\left(R_{2}\right)$.

by applying the heat input at a different point. So, the discrete mode only contains two states, that is the furnace "off", $q_{0}$, and the furnace is "on", $q_{1}$. The continuous dynamics is described as 10

$$
x(t+1)= \begin{cases}\tilde{A}_{0} x(t)+B_{0} u(t)+E_{0} d(t), & q=q_{0} \\ \tilde{A}_{1} x(t)+B_{1} u(t)+E_{1} d(t), & q=q_{1} .\end{cases}
$$

where

$$
\begin{aligned}
& A_{0}^{1}=\left(\begin{array}{cc}
0.825 & 0.135 \\
0.68 & 1
\end{array}\right), A_{0}^{2}=\left(\begin{array}{cc}
1 & 0.35 \\
0.068 & 0.555
\end{array}\right) \\
& B_{0}=\left(\begin{array}{c}
1.8179 \\
0.0773
\end{array}\right), E_{0}=\left(\begin{array}{c}
0.0387 \\
0.3772
\end{array}\right) \\
& A_{1}^{1}=\left(\begin{array}{cc}
-0.664 & 0.199 \\
0.199 & 0.264
\end{array}\right), A_{1}^{2}=\left(\begin{array}{ll}
-0.7 & 0.32 \\
0.32 & 0.44
\end{array}\right) \\
& B_{1}=\left(\begin{array}{c}
0.8101 \\
0.1369
\end{array}\right), E_{1}=\left(\begin{array}{c}
0.1369 \\
0.5363
\end{array}\right)
\end{aligned}
$$

The partition of the state space is obtained by considering the following hyperplane

$$
\begin{aligned}
& h_{1}(x)=x_{1}-20, h_{2}(x)=x_{2}-5 \text {, } \\
& h_{3}(x)=x_{2}, \quad h_{4}(x)=x_{1}
\end{aligned}
$$

Consider region $R_{1}=\left(\left\{q_{0}, q_{1}\right\}, P_{1}\right)$ and $R_{2}=$ $\left(\left\{q_{0}, q_{1}\right\}, P_{2}\right)$, where $P_{1}=\left\{x \in \mathbb{R}^{2} \mid\left(0 \leq x_{1} \leq\right.\right.$ $\left.20) \wedge\left(-20 \leq x_{2} \leq 0\right)\right\}$, and $P_{2}=\left\{x \in \mathbb{R}^{2} \mid(0 \leq\right.$ $\left.\left.x_{1} \leq 20\right) \wedge\left(0 \leq x_{2} \leq 5\right)\right\}$. Our control object is that for every initial sate $\left(q_{0}, x_{0}\right)$ within region $R_{1}$ there exist control $u \in U$ and $\sigma_{c} \in \Sigma_{c}$ so that from $\left(q_{0}, x_{0}\right)$ the state can be driven to $R_{2}$ without entering a third region, then the state will stay inside $R_{2}{ }^{11}$, no matter what the dynamic uncertainty, continuous and discrete disturbances are. Let's check the attainability. We first calculate pre $\left(R_{2}\right)$, which cover the region $R_{2}$, so $R_{2}$ is safe. By recursively using pre(.), we find that $R_{1}$ can be driven to $R_{2}$ in three steps, i.e. reachable. So the attainability of the specification is satisfied. The figure 1 plot the predecessor set $\operatorname{pre}_{c}^{q_{1}}\left(R_{2}\right)$ for illustration.

\section{CONCLUSION}

In this paper, we consider hierarchical control for a class of uncertain piecewise linear hybrid dynamical systems. The existence of a controller such that the closed loop systems follow desired output of exosystem under uncertainty and disturbance is considered. The proof of the controller existence (Koutsoukos, 2000b; Koutsoukos and Antsaklis, 2001) also gives a constructive way for the regulator design. Often, we are also interested in the real-time behavior of a system. For example, we may require that each component is assembled in a manufacturing system in less than $1 \mathrm{~min}$. Real-time specifications can be incorporated into the framework by including explicitly timers in the model of the plant. The timers can be included either in the continuous part using linear oscillators or in the discrete part using finite state machine models.

\section{REFERENCES}

Hespanha, J., D. Liberzon, A. Stephen Morse, Brian D. O. Anderson, Thomas S. Brinsmead and Franky De Bruyne (2001). Multiple model adaptive control. part 2: switching. International Journal of Robust and Nonlinear Control 11(5), 479-496.

Johansson, M. (1999). Piecewise Linear Control Systems. PhD thesis. Lund Institute of Technology.

Koutsoukos, X. and P. Antsaklis (2001). Hierarchical control of piecewise linear hybrid dynamical systems based on discrete abstractions. ISIS Tech. Rept. ISIS-2001001,http://www.nd.edu/ isis/tech.html.

Koutsoukos, X., P. Antsaklis, J. Stiver and M. Lemmon (2000a). Supervisory control of hybrid systems. Proceedings of IEEE 88(7), 1026-1049.

Koutsoukos, X.D. (2000b). Analysis and Design of Piecewise Linear Hybrid Dynamical Systems. PhD thesis. University of Notre Dame.

Motzkin, T. (1952). The theory of linear inequalities. Rand Corp.. Santa Monica, CA.

Raisch, J. and S. O'Young (1998). Discrete approximation and supervisory control of continuous systems. IEEE Tran. on $A C$ 43(4), 568-573.

Sontag, E. (1982). Remarks on piecewise-linear algebra. Pacific Journal of Mathematics 82(1), 180-210.

Sontag, E. (1996). Interconnected automata and linear systems: A theoretical framework in discrete-time. Pacific Journal of Mathematics 1066, 436-448.

Stiver, J., P. Antsaklis and M. Lemmon (1996). A logical des approach to the design of hybrid control systems. Mathl. Comput. Modelling 23(11/12), 55-76.

\footnotetext{
${ }^{10}$ using zero-order hold sampling with $T=1$ s.

${ }^{11}$ Of cause we can build an automata, exosystem, to describe such simple specification.
} 